\title{
Measuring bulk density variations in a moving powder bed via terahertz in-line sensing
}

Sandra Stranzinger ${ }^{\mathrm{a}, \mathrm{b}}$, Eva Faulhammer ${ }^{\mathrm{a}}$, Jingyi $\mathrm{Li}^{\mathrm{c}}$, Runqiao Dong ${ }^{\mathrm{c}}$, Johannes G Khinast ${ }^{\mathrm{a}, \mathrm{b}}$, J Axel Zeitler $^{\mathrm{c}}$, Daniel Markl ${ }^{\mathrm{d}, \mathrm{e} *}$

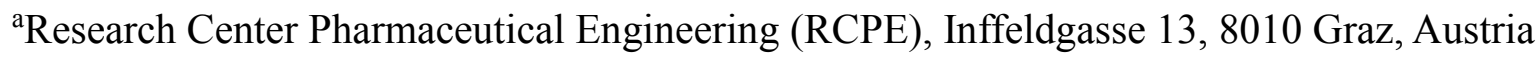

${ }^{\mathrm{b}} \mathrm{Graz}$ University of Technology, Institute for Process and Particle Engineering, Inffeldgasse 13, 8010 Graz, Austria

${ }^{\mathrm{c}}$ Department of Chemical Engineering and Biotechnology, University of Cambridge, Philippa Fawcett Drive, CB3 0AS Cambridge, UK

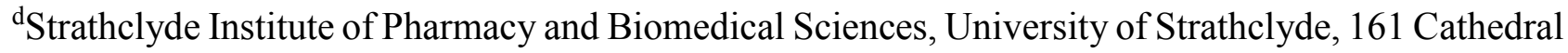
Street, G4 0RE Glasgow, UK

eEPSRC Centre for Innovative Manufacturing in Continuous Manufacturing and Crystallisation, University of Strathclyde, 99 George Street, G1 1RD Glasgow, UK

\begin{abstract}
Monitoring the relative density of static or moving powder inside a process line is essential for manufacturing high-quality products. The aim of this study was to predict density variations in a moving powder bed using terahertz reflection technology. We systematically investigated three grades (varying true density and particle size) of two materials: lactose and silicified microcrystalline cellulose (SMCC). These six powders specifically differ in their compressibility, which can be applied to assess the sensitivity and applicability of our method. The powders were filled into a round container, and terahertz reflection measurements were acquired continuously during the container's rotation. The setup allowed to adjust the relative density by compacting the powders into specific powder bed heights. Each powder was compacted to various relative densities (compression pressures up to $100 \mathrm{kPa}$ ). We calculated the surface refractive index based on the in-line terahertz measurements acquired during rotation, which has a linear dependence on the relative density of the powder. This was confirmed by correlating the refractive index values with the theoretical relative densities based on the bulk and true densities of the powder. The coefficient of determination $\left(R^{2}\right)$ was larger than 0.962 (Lactohale 100) for all six powders, with the highest coefficients for Lactohale $220\left(R^{2}=\right.$ 0.996) and SMCC $50 \mathrm{LD}\left(R^{2}=0.995\right)$. The results suggest that the proposed method can resolve relative densities averaged across the powder bed that are as small as $0.3 \%$ (Lactohale 100). The high acquisition rate of the terahertz system $(15 \mathrm{~Hz})$ made it possible to determine the powder density in 230 positions uniformly distributed throughout the container, facilitating the investigation of the relative density uniformity in the container as a function of the powder bed height. It was observed that SMCC powders undergo a smaller change in the relative density variations upon compaction
\end{abstract}


than the lactose powders. Moreover, the relative density maps clearly indicate local density differences in the powder bed for all powders. The relative density variations (in the horizontal direction) that were introduced by packing of the container prevailed throughout the compaction process for all samples with the exception of Lactohale 220. The presented approach allows a precise resolution of the spatial distribution of relative density, which facilitates an in-depth analysis of powder behavior upon compaction.

Keywords: Powder bulk density, terahertz in-line sensing, refractive index, capsule filling

*Corresponding author: E-mail address: daniel.mark1@strath.ac.uk (Daniel Markl)

\section{Introduction}

Handling granular materials, including powders, plays a crucial role in many industries (such as pharmaceutics, agriculture, food and cosmetic production, mining, catalysis technology, chemical processing and environment) and modern technologies (such as additive manufacturing). In fact, most of the raw materials used for manufacturing end products are granular materials, whose handling (e.g. transporting, conveying, storing, dosing) is known to be challenging due to their applicationdependent performance and their material attributes that hinder an establishment of general constitutive equations relating shear rates and stresses [1-3]. Granular materials, and in particular powders, are considered to be two-phase systems consisting of a dispersed phase of solids of various sizes and gas as the continuous phase. Consequently, the behavior of powder materials depends on the properties of the particle as an individual entity, the properties of an assembly of particles, and the interactions between those assemblies and the continuous phase [4]. Even though bulk properties are known to have an important effect on many processes and unit operations that involve powders and particulates [4], measuring the process/raw material variability in real-time and initiating corrective actions before it can influence the product quality is still a major challenge [5].

This is particularly important for the manufacturing of pharmaceutical solid dosage forms. The production of pharmaceuticals comprises various processes, and the majority of operations (i.e., roller compactors, twin-screw granulators, tableting machines, additive manufacturing and capsule filling) require a densification of the raw or intermediate material. As such, the relative densities of the intermediate and final product are highly critical for the product quality and need to be monitored and controlled to assure a consistent product performance. In general, the powder density can be a useful parameter in the design, optimization and scale-up of manufacturing processes, where density could be used as an equipment-independent scaling parameter [6]. Furthermore, as the pharmaceutical 
industry is moving from batch to continuous processing [7-9], real-time monitoring of the product's quality is inevitable, and robust quality descriptors are essential for realizing the process control [10]. Much of the current literature on the manufacturing of solid dosage forms pays particular attention to techniques for in-line monitoring of the blend uniformity, with near-infrared spectroscopy (NIRS) being the most widely-employed process analyzer [11-17]. Other non-destructive techniques, such as Raman spectroscopy [18-20], light induced fluorescence [21] and chemical imaging [22-25], are used for on- and in-line measuring of the blend uniformity [26]. However, even if the blend uniformity is adequate, density changes in the product may lead to undesirable variations in the product performance. Density variations can either cause an excess/lack of drug in a single dose or affect the microstructure, which may influence the dissolution behavior of the final product [27]. Thus, it is crucial to control not only the drug concentration but also the relative density during processing. To date, only a few studies have attempted to monitor density variations in a powder bed. For example, Singh et al. [5] proposed a novel method for real-time monitoring of powder bulk density using a NIR sensor and performed a sensitivity analysis to quantify the effects of the powder's bulk density on the critical quality attributes of pharmaceutical tablets. Recently, NIRS calibration models for real-time prediction of powder density (tap, bulk and consolidated) were developed for a pharmaceutical formulation [28]. Moreover, other approaches for monitoring powder bulk densities have been reported in the literature: an analytical ultrasound method [29], an air-coupled acoustic technique [30], photo-acoustic testing [31], acoustic emission measurements [32], microwave measurements [33,34], X-ray based methods [35], thermal effusivity monitoring [36] and electrical tomography [37]. However, quantitative real-time measurement of bulk density variations in a moving powder bed remains a challenge [5].

Terahertz time-domain spectroscopy (THz-TDS) has recently emerged as a promising analytical tool for characterizing the pore structure of tablets [38]. In general, within the last ten years, a rapid development of terahertz systems and their commercialization driven by their inherent potential for quality control have created many exciting opportunities in the pharmaceutical sector [39]. The terahertz spectral region, which can be explored using typical commercial systems, covers the range from 0.1 to $4 \mathrm{THz}$ or $3 \mathrm{~mm}$ to $30 \mu \mathrm{m}$, respectively [40]. Compared with NIR, mid-IR and Raman spectroscopy, THz-TDS is inherently less vulnerable to the scattering effects in powders since it operates at a much longer wavelength [38]. Excipients that are most commonly used for the formulation of solid dosage forms are transparent or semi-transparent to terahertz radiation, and a pulse of terahertz radiation can easily penetrate a sample matrix [41]. This makes it possible to perform transmission measurements through tablets with a thicknesses of up to $5.3 \mathrm{~mm}$ [27]. In order to use THz-TDS to quantify the porosity and relative density of powders and porous media, pulses of terahertz radiation are propagated through the material of interest and the pulse delay is used to 
calculate the refractive index of the samples. This information can be combined with an effective medium theory to extract a range of the sample's properties, including pore structure [37,42]. For example, May et al. [43] proposed terahertz pulsed using imaging (TPI) reflection measurements for determining the tablet density, reporting a strong correlation between the TPI results and tablet bulk density and how these relate to the tablet hardness.

This work had two objectives. The first one is the development of a novel approach to quantify density variations in a moving powder bed by means of terahertz technology. In contrast to previous publications, in our case the terahertz probe was applied in the reflection geometry. Powder samples of model excipients, i.e., lactose and silicified microcrystalline cellulose (SMCC) of varying densities and particle sizes, were systematically investigated at various stages of compaction in a moving powder bed. Refractive index values were extracted from the terahertz reflection measurements and the average relative density of the powder in the container was estimated based on the refractive index data. The second part of the paper introduces the method we developed for (1) studying the dependence of relative density on the compression pressure and (2) providing spatially resolved relative density variations in the powder bed for each powder.

\section{Materials and methods}

\subsection{Materials}

Three grades of lactose excipients (Lactohale 100, Lactohale 200, Lactohale 220) supplied by DFE Pharma (Goch, Germany) and three silicified microrystalline cellulose (SMCC) excipients (Prosolv® SMCC 50, Prosolv ${ }^{\circledR}$ SMCC 50 LD, Prosolv ${ }^{\circledR}$ SMCC 90) supplied by JRS Pharma (Rosenberg, Germany) were used as received. In order to minimize the manufacturer and batch-to-batch variations, the experiments were carried out using powders from the same batch and one supplier. Microscope images from a Morphologi G3 Microscope of the six different powders are depicted in Figure 1. 


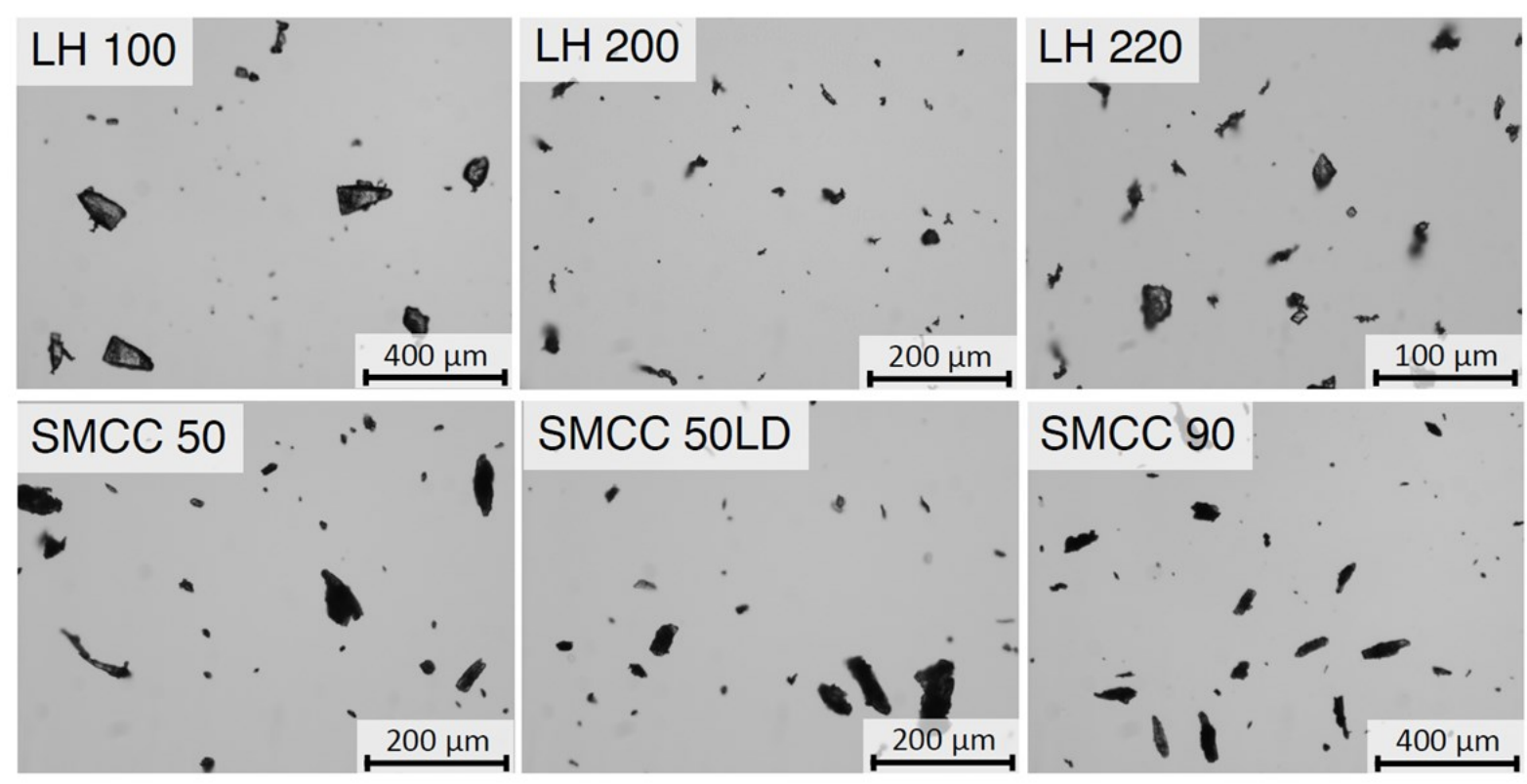

Figure 1: Microscope images of the lactose (top) and SMCC (bottom) excipients.

\subsection{Powder characterization}

All experiments were carried out under controlled environmental conditions $\left(\mathrm{T}=20-24{ }^{\circ} \mathrm{C}, 40-60 \%\right.$ relative humidity). The following material attributes were determined in triplicate: the true density was measured with a helium pycnometer (AccuPac II 1340, Micromeritics, Norcross, USA); the bulk (BD) and tapped density (TD) values and the median particle size (x50) for the lactose excipients were measured previously for the same batch of material $[44,45]$. BD, TD values and the median particle size $(\mathrm{x} 50)$ for SMCC excipients were taken from the technical data sheets (i.e., product sample certificate of analysis) provided by the supplier.

Table 1 presents the bulk powder properties of the excipients and the theoretical amount of powder needed to fill the sample container to an initial target powder layer height of $30 \mathrm{~mm}$. 
Table 1: Densities and particle size (x50) of the lactose and SMCC excipients used in this study. The mass in this Table refers to the theoretical weight of the powder filled in the container, in order to achieve a fill height of $30 \mathrm{~mm}$.

\begin{tabular}{|c|c|c|c|c|c|}
\hline Material & $\begin{array}{c}\text { Bulk density } \\
\left(\mathrm{g} \mathrm{cm}^{-3}\right)\end{array}$ & $\begin{array}{l}\text { Tapped density } \\
\qquad\left(\mathrm{g} \mathrm{cm}^{-3}\right)\end{array}$ & $\begin{array}{l}\text { True density } \\
\qquad\left(\mathrm{g} \mathrm{cm}^{-3}\right)\end{array}$ & $\begin{array}{c}\text { Particle size } \\
\text { x50 }(\mu \mathrm{m})\end{array}$ & $\begin{array}{c}\text { Mass } \\
\text { (g) }\end{array}$ \\
\hline Lactohale 100 & $0.697 \pm 0.004$ & $0.828 \quad \pm 0.013$ & $1.539 \pm 0.003$ & $155.2 \pm 0.6$ & 212.8 \\
\hline Lactohale 200 & \pm 0.003 & \pm 0.002 & $1.543 \pm 0.002$ & $78.7 \pm 0.4$ & 189.9 \\
\hline Lactohale 220 & \pm 0.007 & \pm 0.007 & $1.547 \pm 0.004$ & $13.4 \pm 0.0$ & 122.1 \\
\hline SMCC 50 & $0.31(0.25-0.37)$ & $0.44(0.37-0.50)$ & $1.591 \pm 0.005$ & $64(45-80)$ & 94.7 \\
\hline SMCC 50 LD & $0.24(0.20-0.30)$ & $0.39(0.34-0.47)$ & $1.587 \pm 0.004$ & $50(35-65)$ & 73.3 \\
\hline SMCC 90 & $0.31(0.25-0.37)$ & $0.43(0.37-0.50)$ & $1.585 \pm 0.004$ & $122(90-150)$ & 94.7 \\
\hline
\end{tabular}

All parameters are given as average values \pm SD. The values in brackets for SMCC are the specified limits, as listed in the technical data sheets. The SD for the x 50 values for Lactohale were determined from three repetition runs.

\subsection{Experimental setup}

\subsubsection{Rotary container and compression unit}

The experimental setup, comprising the container, the compression unit, the terahertz fiber-based flexible reflection probe and a motor for rotating the container, is depicted in Figures 2 and 3. For each experimental run, the required amount of powder was manually filled into the container. A scraper was used to smoothen the powder layer prior to measuring the powder layer height. After powder bed preparation, a compression unit as described in detail below, was used to apply pressure to the powder bed. At each compression step, terahertz reflection measurements were continuously acquired during rotation of the container. The copper foil on the outer wall of the container was used as a datum and for reference reflection measurements.

The container was made of high-density polyethylene (HDPE), which is transparent to terahertz radiation. The height of the container's interior was $45 \mathrm{~mm}$. The compression unit consisted of a metal ring with a thickness of $10 \mathrm{~mm}$ combined with a sensor block with a thickness of $63 \mathrm{~mm}$. The pressure in the powder bed was adjusted by tightening a nut on the central screw that was used to assemble the compression unit and the powder container (Figure 2). Since in this study the intended application was a dosator capsule filling process where the powder is compacted to a small extent (i.e. during the dosing step e.g. through pre-compaction, piston compaction), only compression pressures up to 100 $\mathrm{kPa}$ were used. Three load sensors (see Supporting Information) connected to a computer via an Arduino Uno (Arduino, Somerville, US) were used to measure the applied force, which was measured prior to each compaction stage. 
The average relative density, $\bar{\varrho}_{r}$, of the powder was calculated as

$$
\bar{\varrho}_{r}=\frac{m}{V \varrho_{t}}=\frac{m}{A h \varrho_{t}}
$$

where $A$ and $h$ are the cross-section area of the container and the fill height, respectively. $\varrho_{t}$ is the true density (Table 1). $A=2827 \mathrm{~mm}^{2}$ was kept constant throughout all measurements and was determined as

$$
A=\left(\frac{D_{o}-D_{i}}{2}\right)^{2} \pi
$$

with $D_{o}=138 \mathrm{~mm}$ and $D_{i}=78 \mathrm{~mm}$ as the outer and inner diameter of the interior of the container. As mentioned previously, the pressure on the powder bed was adjusted by displacing the compression unit. This displacement was measured in eight positions from the top of the compression unit to the bottom of the container using a digital caliper prior to every measurement. An average value of the displacement was subsequently used to calculate the average fill height, $\bar{h}$, and eventually to determine $\bar{\varrho}_{r}$ (Eq. 1). A motor speed of 4 rpm was applied in each experiment. 


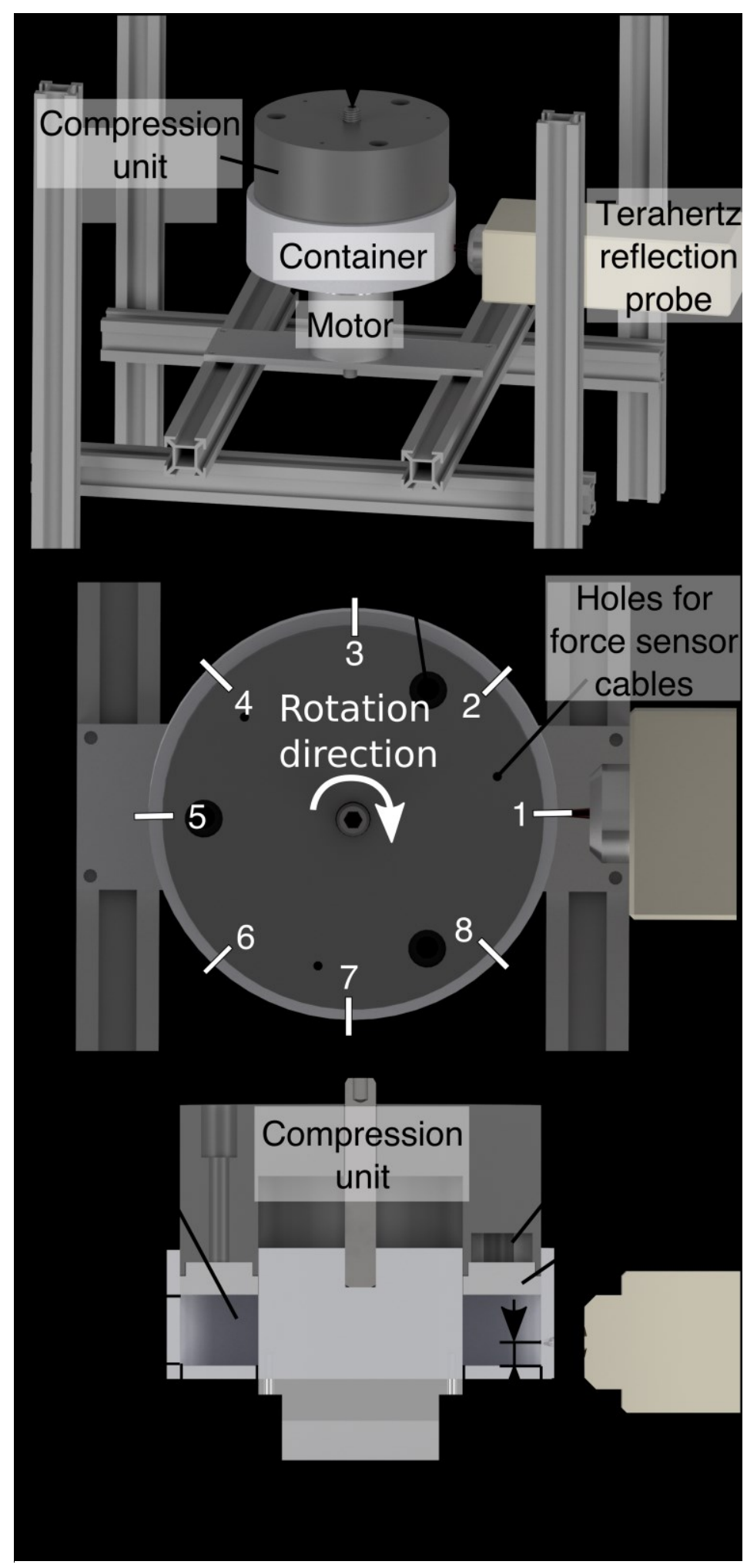

Figure 2: Schematic of the experimental setup for the terahertz reflection measurements. (a) A force is applied to the powder in the container by tightening a nut on the screw. (b) Top view of the setup, indicating the direction of the container's rotation and the displacement measurement positions (labelled as 1 to 8 in red). (c) Cross-section through the center of the container. The interior of container is filled with the powder. The powder bed height is determined in eight different positions, as indicated in (b). 

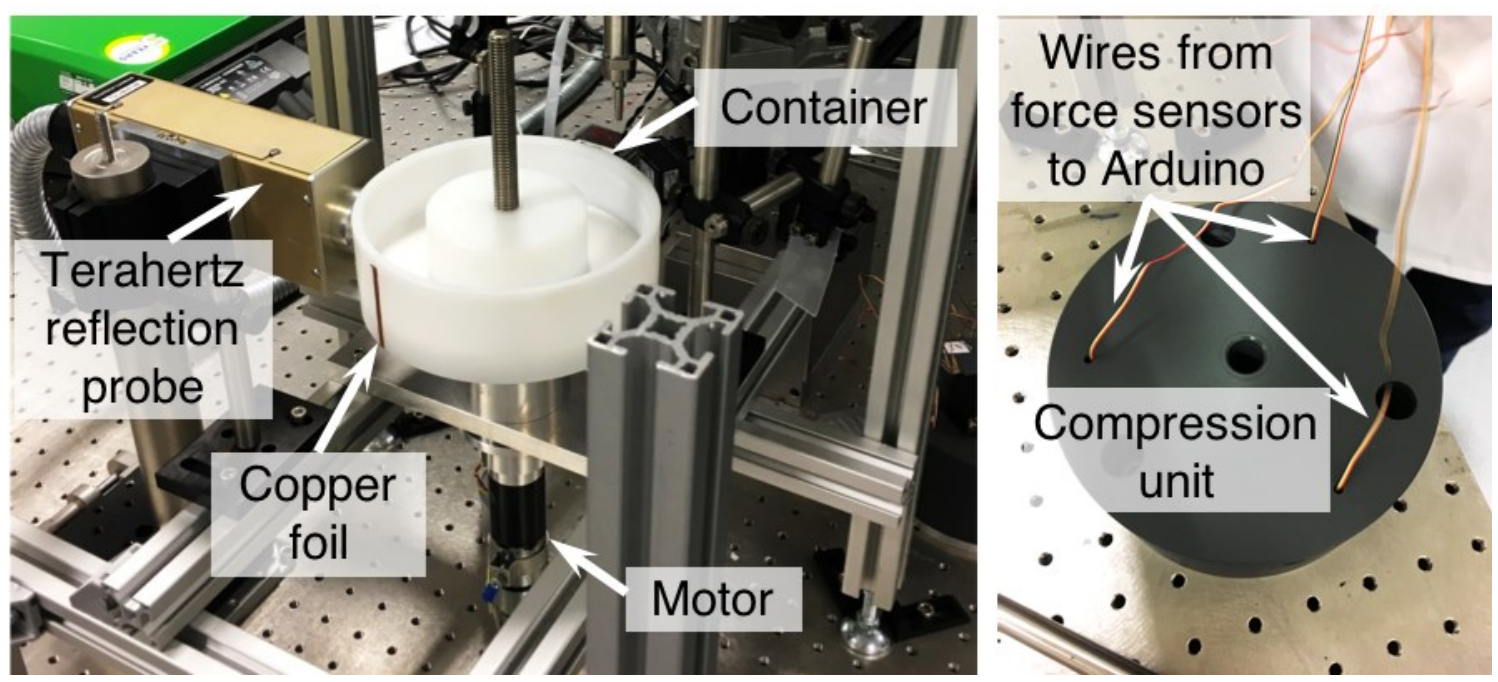

Figure 3: A schematic of the experimental setup for the terahertz reflection measurements. 


\subsubsection{Terahertz pulsed imaging}

Terahertz reflection measurements were continuously acquired for three independent experimental runs per material, and the average relative densities of the different compaction steps in each run were predicted. A commercial time-domain terahertz spectrometer (TeraPulse 4000, TeraView Ltd, Cambridge, UK) coupled with a fiber-based flexible reflection probe was used in this study. The system was configured to acquire the reflected terahertz pulse over an optical time delay of 45 ps at an acquisition rate of $15 \mathrm{~Hz}$. The reflection probe had a silicon lens with a focal length of $18 \mathrm{~mm}$. The beam waist at the focus was $\approx 600 \mu \mathrm{m}$. At each compression step, 2000 terahertz waveforms were acquired continuously while the container was rotating, which yielded a total measurement time of 133 s. These 2000 measurements covered about 8.5 rotations of the container. A narrow strip of copper foil was fixed to the outside of the container to reflect the terahertz pulse and serve as a datum to enable an automatic detection of a full rotation based on the terahertz waveforms. Seven full rotation were averaged, yielding $\mathrm{N}=230$ terahertz waveforms uniformly distributed around the container. The container moved by $1.88 \mathrm{~mm}$ during a single terahertz measurement. The surface refractive index thus represents an average across the probed surface, which is influenced by the circumferential speed of the container and the measurement rate. For example, lowering the motor speed would decrease the probed surface leading to a higher spatial resolution around the container. The focal point was set on the container/powder interface in order to detect the reflected terahertz pulse originating from it. A reduction in the amplitude of the reflected pulse from the container/powder interface related to an increase in the refractive index of the powder, $n_{p}$, which can be calculated using the Fresnel equation for reflection. We would like to emphasize that the calculation of the refractive index based on terahertz reflection measurements differs from determining it via a transmission setup that was employed for studying the porosity of powder compacts $[37,46-49]$.

In order to simplify the calculation of $n_{p}$ based on the reflection measurements, we assumed that the terahertz beam is focused on a normal incidence on the HDPE container and that absorption of the HDPE container and the reflection from the air/container interface are negligible. Although these assumptions introduce a minor systematic error to the absolute magnitude of the calculated $n_{p}$, they do not affect the prediction of relative density based on the terahertz measurements.

In the case of normal incidence of the terahertz beam on the container, the Fresnel reflection coefficient, $r_{c p}$, can be expressed as

$$
r_{c p}=\frac{n_{c}-n_{p}}{n_{c}+n_{p}}
$$

where $n_{c}$ and $n_{p}$ are the refractive indices of the container and the powder, respectively. The refractive index of the HDPE container is $n_{c}=1.54$ [50]. Eq. 3 can then be rearranged as 


$$
n_{p}=\frac{n_{c}\left(r_{c p}+1\right)}{n_{c}-r_{c p}}
$$

$r_{c p}$ was calculated by relating the amplitude of the reflected pulse from the container/powder interface to a reflection from the copper foil on the container. This reference reflection from the copper foil was acquired prior to every experiment. Details of data processing can be found in Supporting Information.

\section{Results}

\subsection{Method development}

\subsubsection{Terahertz reflection measurements}

The terahertz reflection measurements were acquired continuously during the rotation of the container. Since the container does not have a perfectly uniform diameter, the phase of reflection of the container/powder interface varied slightly. Variations due to the container's non-uniformity were corrected by applying the terahertz reflection data for an empty container (see Supporting Information).

In each experimental run, the required amount of powder (as shown in Table 1) was manually filled into the container. A scraper was used to smoothen the powder layer prior to measuring the powder layer height and subsequent mounting the metal ring and the compression unit. As outlined in Section 2.3.1, the powder's relative density was adjusted by displacing the compression unit. For each compaction step the applied pressure on the powder bed was measured using the integrated force sensors. Moreover, a change in the powder layer height was measured at each compaction step with a digital caliper.

The terahertz pulse reflected from the container/powder interface appears negative in the terahertz time-domain waveform (Figure 4) as $n_{c}>n_{p}$. Decreasing the powder bed height results in an increase in $n_{p}$ in the relative density. The difference between the container and the powder refractive indices $\left(\left|n_{c}-n_{p}\right|\right)$ decreases, which reduces the amplitude of the reflected terahertz pulse. In theory, $n_{p}$ ranges from 1 (only air) to the intrinsic refractive index of the powder, i.e., the refractive index of the solid skeleton material without any air voids. The refractive indices of pure $\alpha$-lactose monohydrate and MCC at $1 \mathrm{THz}$ were reported as $\approx 1.67$ [51] and $\approx 1.85$ [48], respectively. However, the intrinsic refractive indices of the powders used in this study may slightly vary from this value. 


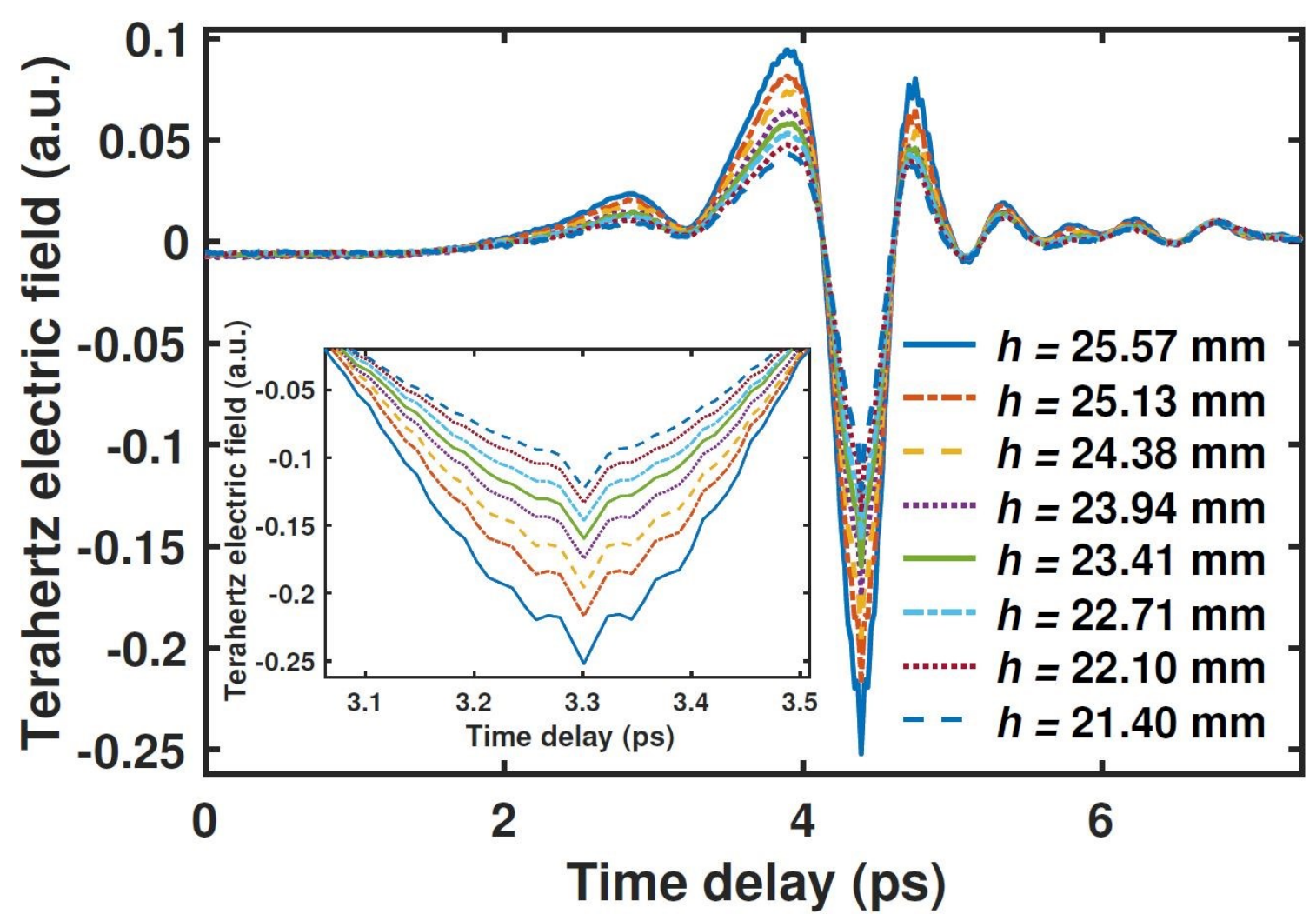

Figure 4: Aligned and averaged terahertz time-domain waveforms for various displacements of the experiment with Lactose 220 at a motor speed of $4 \mathrm{rpm}$.

\subsubsection{Relationship between measured $\boldsymbol{n}_{\boldsymbol{p}}$ and relative density}

As shown in Figure 5, there is a linear relationship between the measured refractive indices and the relative densities of the six powders within the range of relative densities studied. In particular, the refractive index measurements of Lactohale $220\left(R^{2}=0.996\right)$ and SMCC 50 LD $\left(R^{2}=0.995\right)$ correlated strongly with the respective densities (Table 2). These observations are in line with previous studies on the porosity of powder compact, which reported that the refractive index has a linear relationship with the porosity [42]. As such, a linear model was fitted to this calibration data, which is expressed as

$$
\bar{\varrho}_{r}=a_{0}+a_{1} \bar{n}_{p}
$$

Eq. 5

where $a_{0}$ and $a_{1}$ are fitting parameters, $\bar{\varrho}_{r}$ is the average relative density from Eq. 1 and $\bar{n}_{p}=$ $\sum_{i=1}^{N} n_{p, i}$ is the average refractive index with $n_{p, i}$ calculated by using Eq. 4 and the terahertz reflection measurement in position $i$. The dimensions and speed of the container and the acquisition rate of the terahertz system yielded $N=230$ terahertz measurements per container rotation. Position $i$ corresponds to a certain angle in the container (e.g., $i=100$ corresponds to an angle of $157^{\circ}$ ) 
measured from the copper foil in the counter-clockwise direction. The noise was reduced by applying a moving average filter with a size of 5: the amplitude of reflection peak 0 of 5 waveforms was averaged before calculating $n_{p, i}$. The fitting parameters $a_{0}$ and $a_{1}$ were determined for every powder separately. These models were subsequently used to predict the relative densities in each position in the container based on the terahertz reflection measurements.
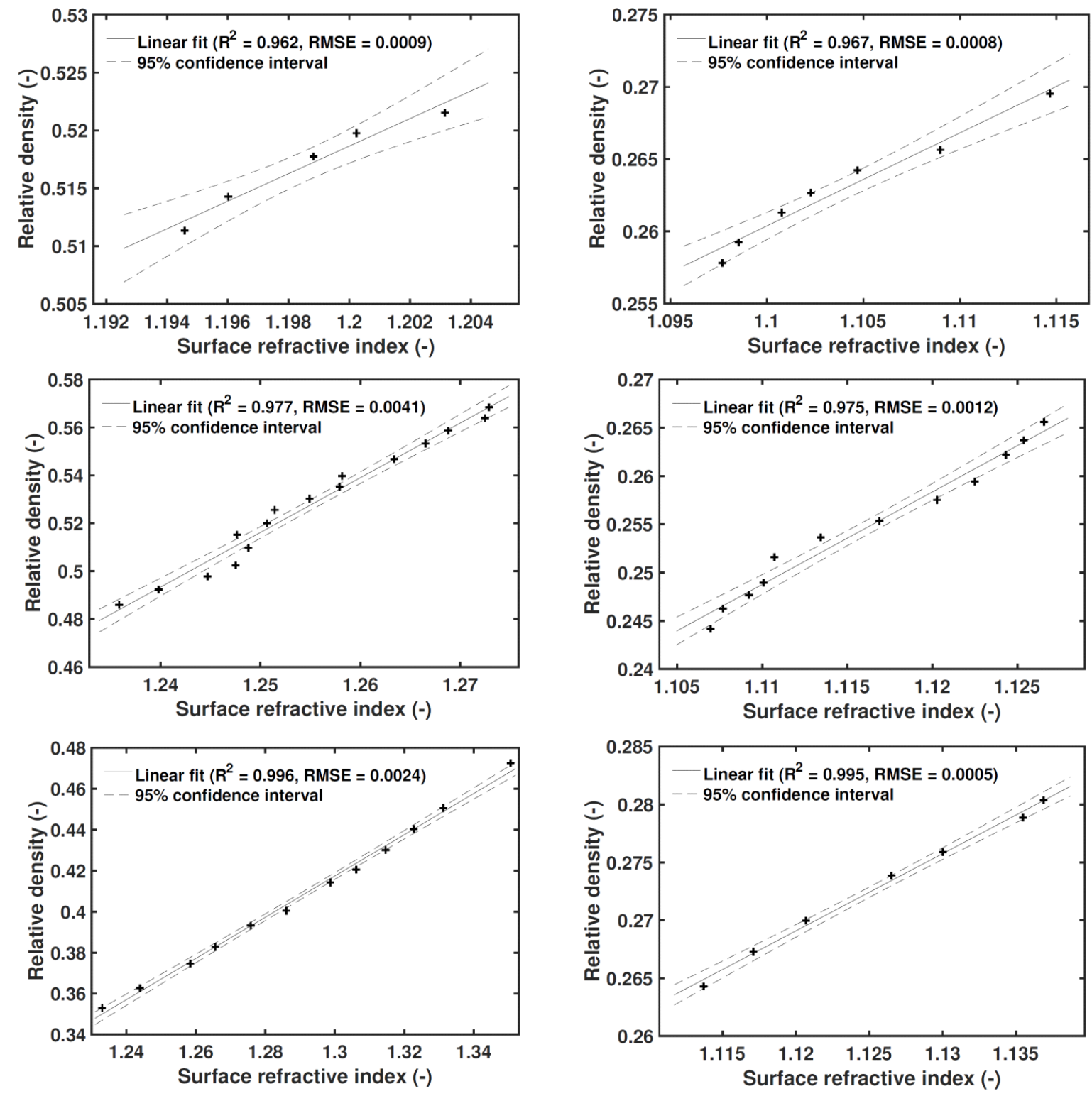

Figure 5: The relative density as a function of surface refractive index calculated based on the terahertz measurements for various powder bed heights. The relative density, $\bar{\varrho}_{r}$, and the refractive index, $\bar{n}_{p}$, were calculated using Eq. 1and Eq. 4, respectively. (a) Lactohale 100, (b) Lactohale 200, (c) Lactohale 220, (d) SMCC 90, (e) SMCC 50 and (f) SMCC 50 LD. 
Table 2: Fitting parameters and assessment of goodness of fit for the various powders.

\begin{tabular}{ccccc}
\hline Material & \multicolumn{2}{c}{ Fitting parameters } & \multicolumn{2}{c}{ Goodness of fit } \\
& $\boldsymbol{a}_{\mathbf{0}}$ & $\boldsymbol{a}_{\mathbf{1}}$ & RMSE & $\boldsymbol{R}^{\mathbf{2}}$ \\
\hline Lactohale 100 & $-0.910 \pm 0.163$ & $1.190 \pm 0.136$ & 0.0009 & 0.962 \\
Lactohale 200 & $-2.336 \pm 0.117$ & $2.281 \pm 0.093$ & 0.0041 & 0.977 \\
Lactohale 220 & $-0.892 \pm 0.026$ & $1.007 \pm 0.020$ & 0.0024 & 0.996 \\
SMCC 50 & $-0.816 \pm 0.054$ & $0.959 \pm 0.049$ & 0.0012 & 0.975 \\
SMCC 50 LD & $-0.478 \pm 0.024$ & $0.667 \pm 0.021$ & 0.0005 & 0.995 \\
SMCC 90 & $-0.447 \pm 0.059$ & $0.643 \pm 0.053$ & 0.0008 & 0.967
\end{tabular}

RMSE: root mean squared error; the fitting parameters are shown with their standard errors.

The range of relative densities varied depending on the powder. The powders in this study have different compressibility values (volume change when compressed under a specific normal force), as demonstrated in our previous study [44]. We reported a compressibility at $8 \mathrm{kPa}$ of $1.05 \%, 12.66 \%$ and 36.95\% for Lactohale 100, Lactohale 200 and Lactohale 220, respectively. This influenced the calibration in that it limited the maximum displacement that could be achieved by the compression unit. In particular, the maximum relative displacement (related to the initial powder bed height) and maximum pressure (in brackets) values were $2 \%(97.7 \mathrm{kPa}), 17 \%(85.5 \mathrm{kPa})$ and $51 \%(72.4 \mathrm{kPa})$ for Lactohale 100, Lactohale 200 and Lactohale 220, respectively. Therefore, the compressibility and the maximum displacement are in good agreement with our previous work, provided that a smaller displacement corresponds to a lower compressibility. The pressure on the powder was calculated based on the measured force using the integrated force sensors.

Given its limited compressibility, it was impossible to significantly vary the relative density of Lactohale 100 in the current experimental setup (Figure 5a). This means that the predictability of the linear model for Lactohale 100 is only valid within a small range of relative densities. The investigation of such small density changes for Lactohale 100, however, indicates that the terahertz method resolved density variations that are as small as $0.3 \%\left(\min _{k \in S}\left(1-\bar{\varrho}_{r}(k) / \bar{\varrho}_{r}(k+1)\right)\right.$, with $S=$ $\{1 \ldots K-1\}$ and $K=$ the number of compression steps). In contrast, given the higher compressibility of Lactohale 200 and Lactohale 220, a much larger range of powder bed heights could be tested, resulting in a broader range of relative densities (Figure 5b-c).

In the preliminary experiments we investigated the effect of various motor speeds on the measurements. The results indicate that the motor speed (in the range of 4 to $16 \mathrm{rpm}$ ) has no influence on the average surface refractive index. However, a faster rotation of the container causes a lower spatial resolution of the terahertz measurements in the container since $N$ decreases with an increasing motor speed at a fixed acquisition rate. As such, we applied a motor speed of $4 \mathrm{rpm}$ in all experiments, 
which resulted in a spatial resolution of $1.57^{\circ}$.

\subsection{Predicting relative density based on terahertz reflection measurements}

The relative density variations of the powder bed were studied via the linear models, as discussed in Section 3.1.2. The relative density was subsequently determined based on the linear model (Eq. 5) as a function of the container angle and the powder bed height.

\subsubsection{Dependence of relative density on the compression pressure}

Data acquired by the force sensors (see Section 2.3.1) were used to develop an in-depth analysis of the relationship between the pressure applied and the relative densities predicted for the two powder types, i.e., lactose and SMCC excipients. Data in Figure 6 suggest that, due to the powders' different compressibility, the range of relative density that can be explored in the rig varies between the two excipients. A broader relative density range for the lactose excipients compared to that of the SMCC excipients is attributed to differences in the compaction behavior, which is significantly affected by the physicochemical and mechanical properties of the material [52]. Heckel [52] proposed densitycompression force relationship, the Heckel plot analysis, which is often used to describe the plasticity of materials. For example, Teixeira [53] reported that, compared to lactose excipients, MCC excipients have a greater plasticity or plastic deformation at relatively low pressure and a lower brittle fraction. Lactose is commonly regarded as a predominately brittle material that breaks easily at relatively low deformation [54], whereas MCC is considered relatively ductile material that can undergo a significantly higher elastic and plastic deformation [54]. In previous studies [44,45], the three used grades of lactose excipients (Lactohale 100, Lactohale 200, Lactohale 220) were extensively characterized, including their bulk powder properties, flow properties and friction properties. In regard to MCC it is important to note that in our study silicified MCC (SMCC), a material produced by a process of 'silicification', was used. Tobyn et al. [55] reported no discernible chemical or polymorphic differences between the tested MCC 90 and SMCC 90, indicating that the 'silicification' process produces a material which is chemically and physically very similar to standard MCC. In terms of surface texturization, scanning electron microscopy revealed distinct differences between the morphological characteristics of MCC 90 and SMCC 90. The latter shows an extensive surface texturization which was conjectured, from the dimensions and shape of the textural features, to be individual or agglomerated colloidal silica particles. These textural differences were only observed in high resolution images and therefore the authors concluded that the silicification does not induce gross changes in the shape and texture of MCC particles. 

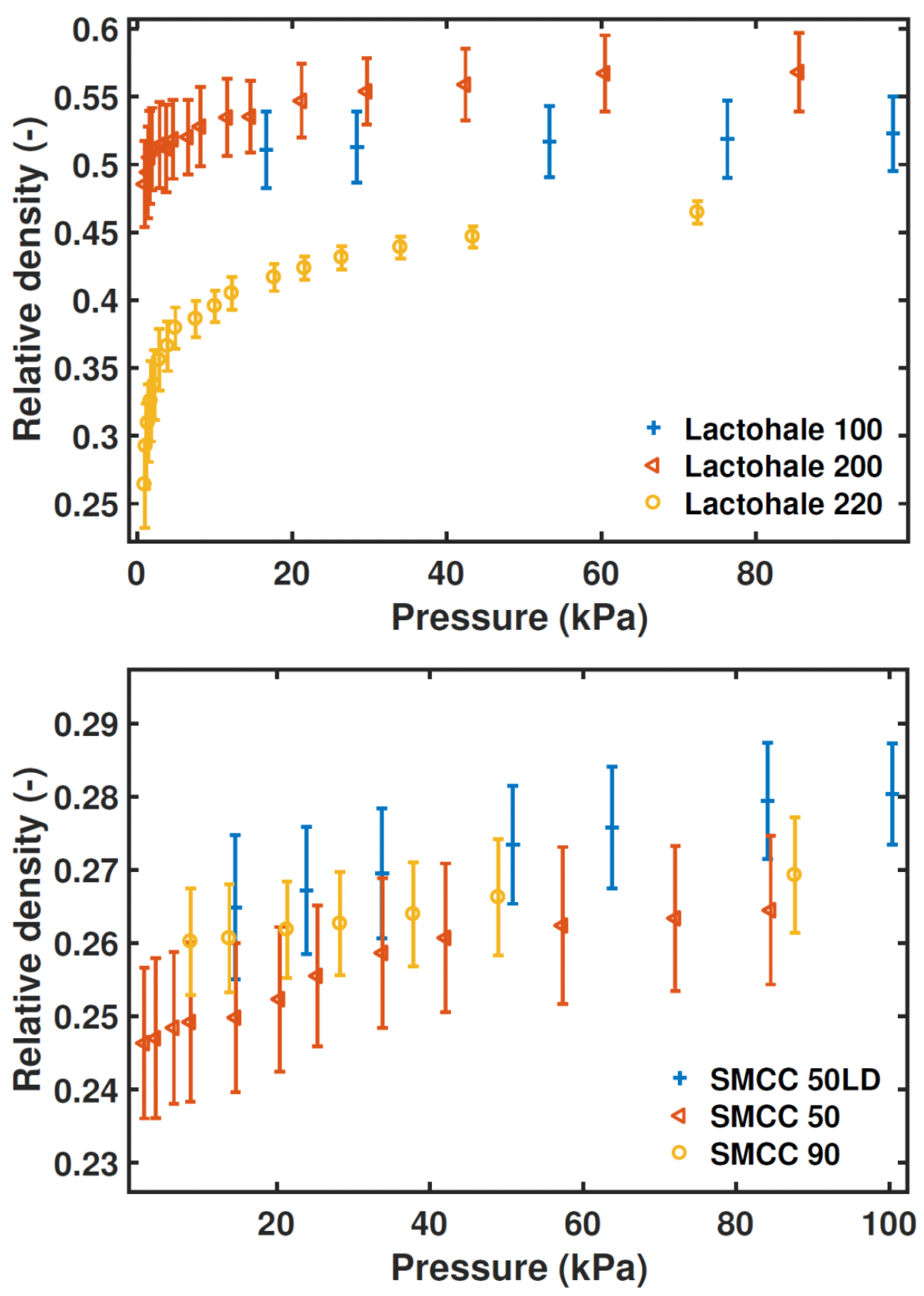

Figure 6: The relative density (predicted from the terahertz measurements) as a function of increasing pressure applied to the powder bed. The standard deviation corresponds to the variation of the powder around the container and was calculated from $\mathrm{N}=230$ terahertz measurements uniformly distributed around the container. (a) lactose excipients (Lactohale 100, Lactohale 200, Lactohale 220); (b) silicified microrystalline cellulose (SMCC) excipients (SMCC 90, SMCC 50, SMCC 50 LD). 


\subsubsection{Spatially-resolved relative density variations}

As described in the previous section, in this setup we were able to spatially resolve relative density variations in the container. Moreover, a precise measurement of the powder bed height in eight positions (see Figure $2 b$ ) of the container facilitates the investigation of density variations and powder bed height variations at a specific angle of rotation. The resulting maps of relative density and powder bed height variation resolved via the angle of rotation for each powder are shown in Figure 7 and Figure 8 for lactose and SMCC, respectively.

Please note that the prediction of relative density values for Lactohale 100 was only calibrated over a small density range $(51-52 \%)$ and the values outside this range were determined via a linear extrapolation. However, despite this limitation, significant density variations were clearly established in the powder bed for Lactohale 100 (Figure 7a). Comparing these local density variations as a function of container angle with the measured powder bed height variations (Figure 7d) makes a relationship between those two parameters evident. In theory, a large powder bed height (blue in Figure 7d-f and in Figure 8d-f) results in a low relative density (blue in Figure 7a-c and Figure 8a-c). This was observed for all powders except for Lactohale 220, which has the largest compressibility factor. Since the terahertz reflection measurement is performed about $9 \mathrm{~mm}$ above the bottom surface of the container, the powder bed height variations for Lactohale 220 are compensated for by the powder between the position of the terahertz reflection measurement and the top surface of the powder bed. This also suggests that the powder bed density varies significantly in the vertical direction. This could be investigated in the future by acquiring terahertz reflection measurements in various vertical positions at each compression step.

The data for Lactohale 100 indicate that applying normal stress to the powder bed in order to decrease the powder bed height does not affect the homogeneity of the powder bed, as reflected by the horizontal color distribution along vertical direction. Moreover, we compared local density variations for Lactohale 200 with those for Lactohale 100, which is a more non-uniform density powder bed upon compaction (Figure $7 \mathrm{~b}$ and e). The results suggest that significant density variations in the container (in the horizontal direction) are introduced when the bed is packed and remain there throughout the compaction process. Based on this observation, we conclude that the filling process for such powders is particularly crucial since an initial variation in the powder bed height will yield a non-uniform relative density distribution even after several compression steps. In contrast, Lactohale 220 has several stages of powder bed variations during compaction. In the beginning, moderate relative density variations in the horizontal direction were detected, which increased upon compaction and decrease again at the smallest powder bed height (Figure 7c and f). The reason may be that this highly cohesive powder has different stages of particle reorganization upon compaction. Moreover, the findings are possibly linked to a significant amount of air entrapped in the powder 
layer, which may introduce inhomogeneity and variations in the relative densities throughout compaction.
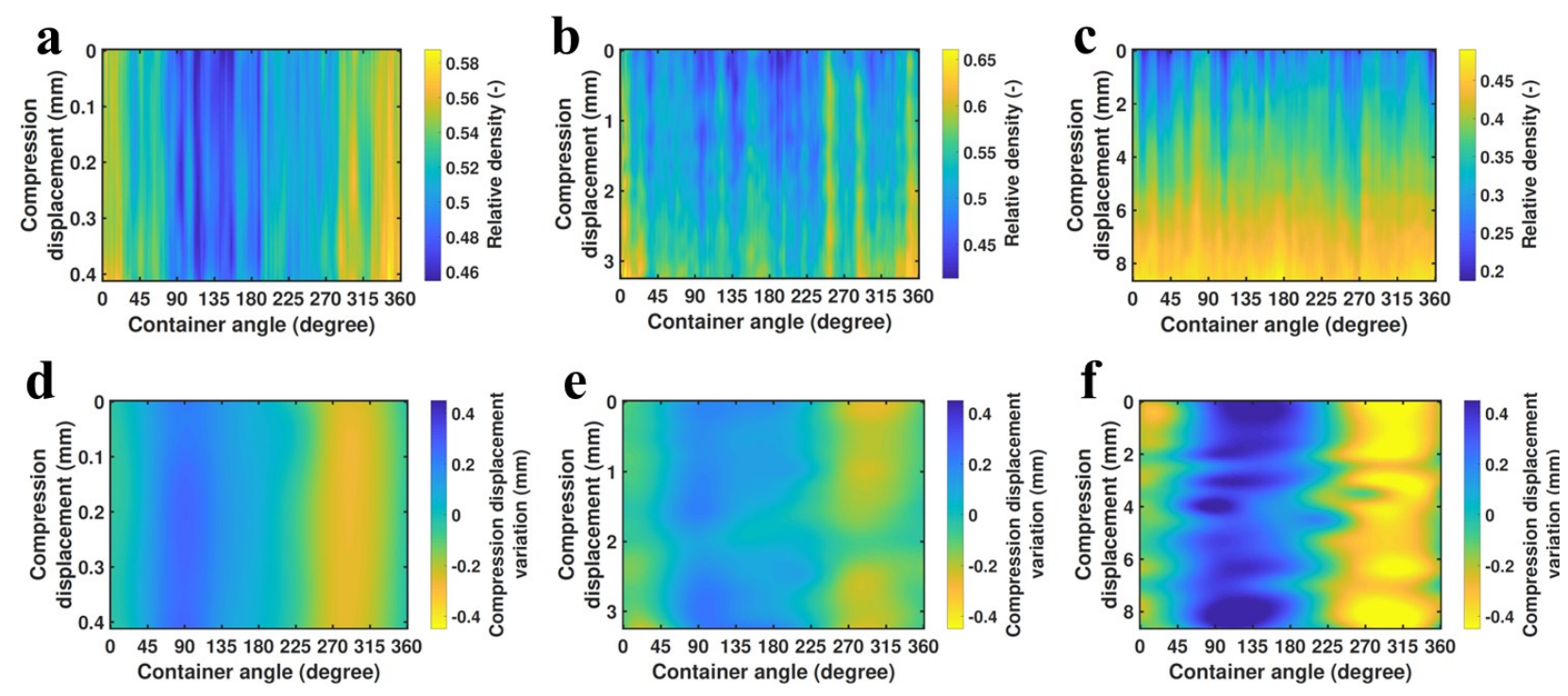

Figure 1: Maps of relative density distributions (a-c) and powder bed height variations (d-e) as a function of average powder bed height, $\bar{h}$, and the angle on the container. (a,d) Lactohale 100, (b,e) Lactohale 200, (c,f) Lactohale 220. The data were interpolated using a cubic interpolation. The compression displacement represents the change of powder bed height from its initial height.

Compared to lactose, SMCC excipients undergo a smaller change in the relative density variations, but have a notable local density differences within the powder bed. The predicted relative density variations are reflected by variations in the powder bed height for all SMCC materials. The resulting maps of relative density variations (at the top half of Figure 8) clearly show similarities between SMCC 90 (Figure 8c) and SMCC 50 LD (Figure 8b). This result is rather surprising if we consider the different material attributes of those powders, e.g., the particle size. Since the particle size, along with the particle shape, elasticity, plasticity and brittleness, is known to affect the strength of bonds and arrangements of particles in a tablet [27,56], we would expect similar distribution maps for SMCC 50 (Figure 8a) and SMCC 50 LD (Figure 8b). However, if one considers the bulk densities of the two powders, SMCC 50 and SMCC 50 LD, the latter has a lower bulk density, as also evident from the sample label "LD" (low density). It is well known that a lower density (higher porosity) may facilitate compressibility, i.e., the densification of a powder bed due to the application of a stress [57]. Therefore, a higher relative density (more precisely local areas with higher relative density) observed upon compression of the powder bed for the SMCC 50 LD (see Figure 8b) may be attributed to differences in the bulk densities rather than in the particle sizes. These findings are also reflected in the resulting maps of powder bed height variations (Figure 8d-f), which confirm the presence of local density variations in the powder bed. 

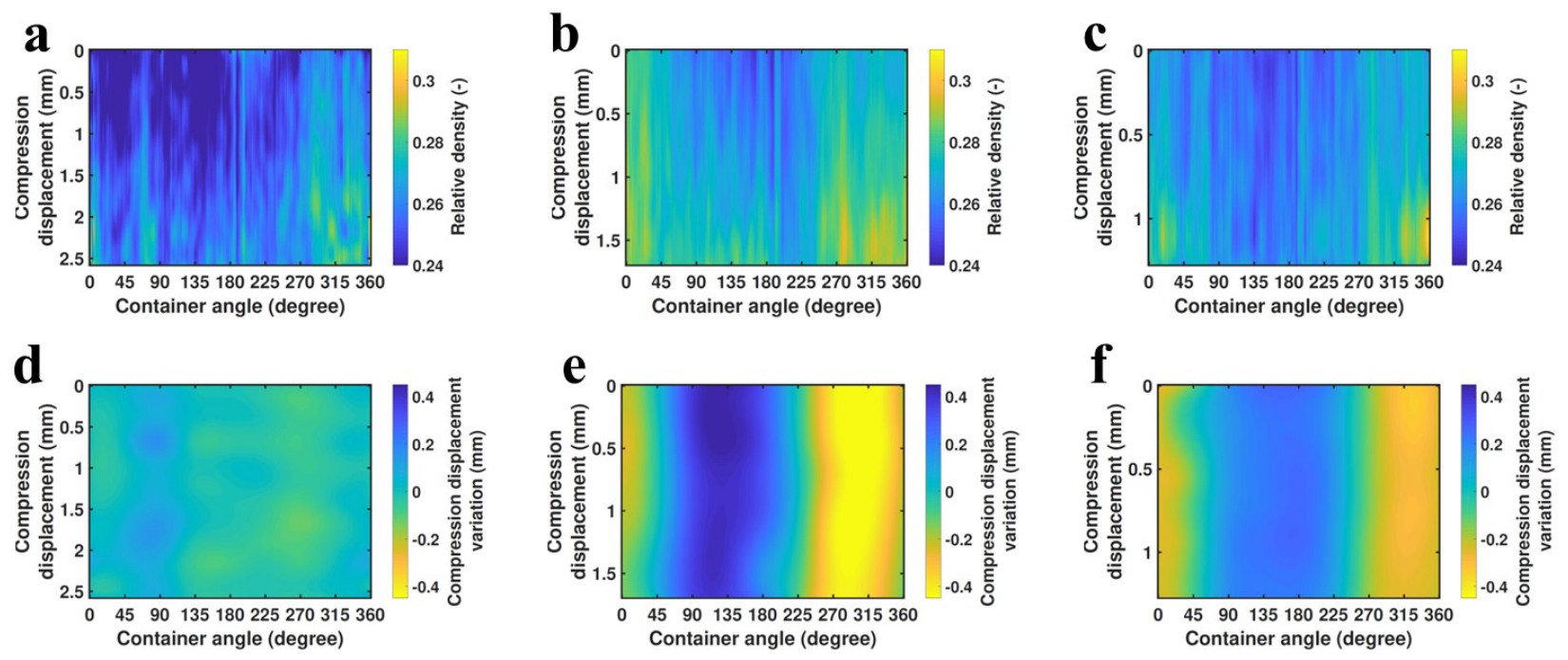

Figure 2: Maps of relative density distributions (a-c) and powder bed height variations (d-e) as a function of average powder bed height, $\bar{h}$, and angle on the container. (a,d) SMCC 50, (b,e) SMCC $50 \mathrm{LD}$, (c,f) SMCC 90. The compression displacement represents the change of powder bed height from its initial height.

\section{Conclusions}

This study presents an analysis of powder density variations in a rotating container via terahertz reflection measurements. The ability to spatially resolve density variations in a powder bed is relevant for a wide range of industrial applications, processes and unit operations, and especially for processes in which density variations of the bulk powder can result in out-of-spec end products. In the case of tableting process, the proposed method would facilitate the development of an in-depth understanding of the effect of compaction force and compression speed on the density variations and the porosity of a tablet, which is known to be one of the most important contributors to the disintegration of immediate-release tablets [56]. Another application example is a direct measurement of powder density variations during capsule filling. In particular, the method could be adjusted for implementation as an in-line process analyzer to determine the bulk density variations inside a dosator nozzle when the powder is collected from a moving powder bed and transferred into a capsule. Establishing a correlation between the bulk density inside the nozzle and the resulting fill weight and fill weight variation of filled capsules, which are known as critical quality attributes [58], would enhance process understanding and lead to process optimization. Although real-time measurement of density variations remains a challenge, it is highly desired to enhance the product quality [5]. Overall, our method has great potential to quantitatively measure bulk density variations in static or moving powder bulk/powder streams and can be implemented as a process analyzer to continuously control the material variations during a batch or continuous manufacturing process (in such industries as 
pharma, food, cosmetic, etc.).

\section{Acknowledgments}

This work has been funded by the Austrian COMET Program under the auspices of the Austrian Federal Ministry of Transport, Innovation and Technology (bmvit), the Austrian Federal Ministry of Economy, Family and Youth (bmwfj) and by the State of Styria (Styrian Funding Agency SFG). COMET is managed by the Austrian Research Promotion Agency FFG. The authors would like to thank MG2 for the scientific and financial support. Furthermore, the authors thank JRS Pharma (Rosenberg, Germany) for providing SMCC excipients and DFE Pharma (Goch, Germany) for providing the lactose excipients. Supporting information is provided electronically with this article online.

\section{References}

[1] D. Schulze, Powders and bulk solids: behavior, characterization, storage and flow, Springer Berlin Heidelberg, 2007.

[2] M. Stiess, Mechanische Verfahrenstechnik -Partikeltechnologie 1, Springer Berlin Heidelberg, 2008.

[3] J. Duran, Sands, powders, and grains: an introduction to the physics of granular materials, Springer Science+Business Media New York, 2012.

[4] E. Ortega-Rivas, Bulk properties of food particulate materials: An appraisal of their characterisation and relevance in processing, Food Bioprocess Technol. 2 (2009) 28-44.

[5] R. Singh, A.D. Román-Ospino, R.J. Romañach, M. Ierapetritou, R. Ramachandran, Real time monitoring of powder blend bulk density for coupled feed-forward/feed-back control of a continuous direct compaction tablet manufacturing process, Int. J. Pharm. 495 (2015) 612-625.

[6] B.C. Hancock, J.T. Colvin, M.P. Mullarney, A.V. Zinchuk, The relative densities of pharmaceutical powders, blends, dry granulations and immediate-release tablets, Pharm. Technol. 27 (2003) 64-80.

[7] K. Plumb, Continuous processing in the pharmaceutical industry, Chem. Eng. Res. Des. 83 (2005) $730-738$.

[8] S. Buchholz, Future manufacturing approaches in the chemical and pharmaceutical industry, Chem. Eng. Process. Process Intensif. 49 (2010) 993-995.

[9] P. Poechlauer, J. Manley, R. Broxterman, B. Gregertsen, M. Ridemark, Continuous processing in the manufacture of active pharmaceutical ingredients and finished dosage forms: an industry perspective, Org. Process Res. Dev. 16 (2012) 1586-1590.

[10] A.U. Vanarase, M. Alcalà, J.I. Jerez Rozo, F.J. Muzzio, R.J. Romañach, Real-time monitoring of drug concentration in a continuous powder mixing process using NIR spectroscopy, Chem. Eng. Sci. 65 (2010) 5728-5733. 
[11] A.S. El-Hagrasy, F. D’Amico, J.K. Drennen, A process analytical technology approach to nearinfrared process control of pharmaceutical powder blending. Part I: D-optimal design for characterization of powder mixing and preliminary spectral data evaluation, J. Pharm. Sci. 95 (2006) 392-406.

[12] Y. Lin, W. Li, J. Xu, P. Boulas, Development of a NIR-based blend uniformity method for a drug product containing multiple structurally similar actives by using the quality by design principles, Int. J. Pharm. 488 (2015) 120-126.

[13] C.C. Corredor, R. Lozano, X. Bu, R. McCann, J. Dougherty, T. Stevens, D. Both, P. Shah, Analytical method quality by design for an on-line near-infrared method to monitor blend potency and uniformity, J. Pharm. Innov. 10 (2014) 47-55.

[14] W. Li, H. Qu, Characterization of herbal powder blends homogeneity using near-infrared spectroscopy, J. Innov. Opt. Health Sci. 7 (2014) 1450004.

[15] W. Li, M.C. Johnson, R. Bruce, S. Ulrich, H. Rasmussen, G.D. Worosila, Mass-balanced blend uniformity analysis of pharmaceutical powders by at-line near-infrared spectroscopy with a fiberoptic probe, Int. J. Pharm. 326 (2006) 182-185.

[16] J.J. Moes, M.M. Ruijken, E. Gout, H.W. Frijlink, M.I. Ugwoke, Application of process analytical technology in tablet process development using NIR spectroscopy: Blend uniformity, content uniformity and coating thickness measurements, Int. J. Pharm. 357 (2008) 108-118.

[17] G. Reich, Near-infrared spectroscopy and imaging: Basic principles and pharmaceutical applications, Adv. Drug Deliv. Rev. 57 (2005) 1109-1143.

[18] D.S. Hausman, R.T. Cambron, A. Sakr, Application of Raman spectroscopy for on-line monitoring of low dose blend uniformity, Int. J. Pharm. 298 (2005) 80-90.

[19] J. Arruabarrena, J. Coello, S. Maspoch, Raman spectroscopy as a complementary tool to assess the content uniformity of dosage units in break-scored warfarin tablets, Int. J. Pharm. 465 (2014) 299-305.

[20] H. Wikström, S. Romero-Torres, S. Wongweragiat, J.A.S. Williams, E.R. Grant, L.S. Taylor, On-line content uniformity determination of tablets using low-resolution Raman spectroscopy, Appl. Spectrosc. 60 (2006) 672-681.

[21] V. Karumanchi, M.K. Taylor, K.J. Ely, W.C. Stagner, Monitoring powder blend homogeneity using light-induced fluorescence, AAPS PharmSciTech. 12 (2011) 1031-1037.

[22] J.G. Osorio, G. Stuessy, G.J. Kemeny, F.J. Muzzio, Characterization of pharmaceutical powder blends using in situ near-infrared chemical imaging, Chem. Eng. Sci. 108 (2014) 244-257.

[23] H. Ma, C.A. Anderson, Characterization of pharmaceutical powder blends by NIR chemical imaging, J. Pharm. Sci. 97 (2008) 3305-3320.

[24] R.C. Lyon, D.S. Lester, E.N. Lewis, E. Lee, L.X. Yu, E.H. Jefferson, A.S. Hussain, Near-infrared spectral imaging for quality assurance of pharmaceutical products: analysis of tablets to assess powder blend homogeneity., AAPS PharmSciTech. 3 (2002) E17.

[25] A.S. El-Hagrasy, H.R. Morris, F. D’Amico, R.A. Lodder, J.K. Drennen, Near-infrared spectroscopy and imaging for the monitoring of powder blend homogeneity, J. Pharm. Sci. 90 (2001) 1298-1307. 
[26] H. Wang, D. Barona, S. Oladepo, L. Williams, S. Hoe, D. Lechuga-Ballesteros, R. Vehring, Macro-Raman spectroscopy for bulk composition and homogeneity analysis of multi-component pharmaceutical powders, J. Pharm. Biomed. Anal. 141 (2017) 180-191.

[27] D. Markl, J. Sauerwein, D.J. Goodwin, S. van den Ban, J.A. Zeitler, Non-destructive determination of disintegration time and dissolution in immediate release tablets by terahertz transmission measurements, Pharm. Res. 34 (2017) 1012-1022.

[28] A.D. Román-Ospino, R. Singh, M. Ierapetritou, R. Ramachandran, R. Méndez, C. OrtegaZuñiga, F.J. Muzzio, R.J. Romañach, Near infrared spectroscopic calibration models for real time monitoring of powder density, Int. J. Pharm. 512 (2016) 61-74.

[29] J.T.T. Leskinen, S.P. Simonaho, M. Hakulinen, J. Ketolainen, In-line ultrasound measurement system for detecting tablet integrity, Int. J. Pharm. 400 (2010) 104-113.

[30] I. Akseli, D. Dey, C. Cetinkaya, Mechanical property characterization of bilayered tablets using nondestructive air-coupled acoustics., AAPS PharmSciTech. 11 (2010) 90-102.

[31] J. Ketolainen, M. Oksanen, J. Rantala, J. Stor-Pellinen, M. Luukkala, P. Paronen, Photoacoustic evaluation of elasticity and integrity of pharmaceutical tablets, Int. J. Pharm. 125 (1995) 45-53.

[32] A. Hakanen, E. Laine, Acoustic characterization of a microcrystalline cellulose powder during and after its compression, Drug Dev. Ind. Pharm. 21 (1995) 1573-1582.

[33] S. Trabelsi, A.W. Kraszewski, S.O. Nelson, A microwave method for on-line determination of bulk density and moisture content of particulate materials, IEEE Trans. Instrum. Meas. 47 (1998) 127-132.

[34] S. Trabelsi, S.O. Nelson, Calibration methods for nondestructive microwave sensing of moisture content and bulk density of granular materials, Trans. ASAE. 47 (2004) 1999.

[35] I. Akseli, S. Iyer, H.P. Lee, A.M. Cuitiño, A quantitative correlation of the effect of density distributions in roller-compacted ribbons on the mechanical properties of tablets using ultrasonics and x-ray tomography, AAPS PharmSciTech. 12 (2011) 834-853.

[36] M.K. Ghorab, R. Chatlapalli, S. Hasan, A. Nagi, Application of thermal effusivity as a process analytical technology tool for monitoring and control of the roller compaction process., AAPS PharmSciTech. 8 (2007) 23.

[37] C.E. Davies, S.J. Tallon, N. Brown, Continuous monitoring of bulk density and particle size in flowable powders and grains, Chem. Eng. Res. Des. 83 (2005) 782-787.

[38] D. Markl, P. Wang, C. Ridgway, A.P. Karttunen, M. Chakraborty, P. Bawuah, P. Pääkkönen, P. Gane, J. Ketolainen, K.E. Peiponen, J.A. Zeitler, Characterization of the pore structure of functionalized calcium carbonate tablets by terahertz time-domain spectroscopy and $\mathrm{x}$-ray computed microtomography, J. Pharm. Sci. 106 (2017) 1586-1595.

[39] Y.C. Shen, Terahertz pulsed spectroscopy and imaging for pharmaceutical applications: A review, Int. J. Pharm. 417 (2011) 48-60.

[40] E. Bründermann, H.-W. Hübers, M.F. Kimmitt, Terahertz techniques, Springer, 2012.

[41] J.A. Zeitler, Y. Shen, C. Baker, P.F. Taday, M. Pepper, T. Rades, Analysis of coating structures and interfaces in solid oral dosage forms by three dimensional terahertz pulsed imaging, J. Pharm. Sci. 96 (2007) 330-340. 
[42] D. Markl, A. Strobel, R. Schlossnikl, J. Bøtker, P. Bawuah, C. Ridgway, J. Rantanen, T. Rades, P. Gane, K.-E. Peiponen, J.A. Zeitler, Characterisation of pore structures of pharmaceutical tablets: a review, Int. J. Pharm. 538 (2018) 188-214.

[43] R.K. May, K.E. Su, L. Han, S. Zhong, J.A. Elliott, L.F. Gladden, M. Evans, Y. Shen, J.A. Zeitler, Hardness and density distributions of pharmaceutical tablets measured by terahertz pulsed imaging, J. Pharm. Sci. 102 (2013) 2179-2186.

[44] S. Stranzinger, E. Faulhammer, V. Calzolari, S. Biserni, R. Dreu, R. Š, A. Paudel, J.G. Khinast, The effect of material attributes and process parameters on the powder bed uniformity during a low-dose dosator capsule filling process, Int. J. Pharm. 516 (2017) 9-20.

[45] S. Stranzinger, E. Faulhammer, O. Scheibelhofer, V. Calzolari, S. Biserni, A. Paudel, J.G. Khinast, Study of a Low-dose Capsule Filling Process by Dynamic and Static Tests for Advanced Process Understanding, Int. J. Pharm. 540 (2018) 22-30.

[46] P. Bawuah, M. Chakraborty, T. Ervasti, J.A. Zeitler, J. Ketolainen, P.A.C. Gane, K.E. Peiponen, A structure parameter for porous pharmaceutical tablets obtained with the aid of Wiener bounds for effective permittivity and terahertz time-delay measurement, Int. J. Pharm. 506 (2016) 8792.

[47] P. Bawuah, T. Ervasti, N. Tan, J.A. Zeitler, J. Ketolainen, K.E. Peiponen, Noninvasive porosity measurement of biconvex tablets using terahertz pulses, Int. J. Pharm. 509 (2016) 439-443.

[48] P. Bawuah, N. Tan, S.N.A. Tweneboah, T. Ervasti, J. Axel Zeitler, J. Ketolainen, K.E. Peiponen, Terahertz study on porosity and mass fraction of active pharmaceutical ingredient of pharmaceutical tablets, Eur. J. Pharm. Biopharm. 105 (2016) 122-133.

[49] C. Ridgway, P. Bawuah, D. Markl, J.A. Zeitler, J. Ketolainen, K.E. Peiponen, P. Gane, On the role of API in determining porosity, pore structure and bulk modulus of the skeletal material in pharmaceutical tablets formed with MCC as sole excipient, Int. J. Pharm. 526 (2017) 321-331.

[50] S. Wietzke, C. Jansen, M. Reuter, T. Jung, D. Kraft, S. Chatterjee, B.M. Fischer, M. Koch, Terahertz spectroscopy on polymers: a review of morphological studies, J. Mol. Struct. 1006 (2011) 41-51.

[51] E.P. Parrott, J.A. Zeitler, L.F. Gladden, Accurate determination of optical coefficients from chemical samples using terahertz time-domain spectroscopy and effective medium theory, Opt. Lett. 34 (2009) 3722-3724.

[52] R.W. Heckel, An analysis of powder compaction phenomena, Trans. Met. Soc. AIME. 221 (1961) 1001-1008.

[53] A.Z.A. Teixeira, Compaction characteristics of the powder from the seed coat of Tingui (Magonia pubescens), Estud. Biol. 29 (2007) 277-282.

[54] G. Alderborn, C. Nystrom, Pharmaceutical powder compaction technology, Marcel Dekker, Inc., 1996.

[55] M.J. Tobyn, G.P. McCarthy, J.N. Staniforth, S. Edge, Physicochemical comparison between microcrystalline cellulose and silicified microcrystalline cellulose, Int. J. Pharm. 169 (1998) 183-194.

[56] D. Markl, J.A. Zeitler, A review of disintegration mechanisms and measurement techniques, Pharm. Res. 34 (2017) 890-917. 
[57] S. Patel, A.M. Kaushal, A.K. Bansal, Compression physics in the formulation development of tablets, Crit. Rev. Ther. Drug Carr. Syst. 23 (2006).

[58] E. Faulhammer, M. Llusa, C. Radeke, O. Scheibelhofer, S. Lawrence, S. Biserni, V. Calzolari, J.G. Khinast, The effects of material attributes on capsule fill weight and weight variability in dosator nozzle machines, Int. J. Pharm. 471 (2014) 332-338. 